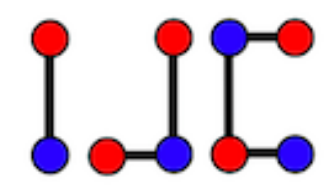

\title{
On size multipartite Ramsey numbers for stars
}

\author{
Anie Lusiani $^{\mathrm{a}}$, Edy Tri Baskoro ${ }^{\mathrm{b}}$, Suhadi Wido Saputro ${ }^{\mathrm{b}}$ \\ ${ }^{a}$ Politeknik Negeri Bandung, Indonesia \\ ${ }^{b}$ Institut Teknologi Bandung, Indonesia \\ anie.lusiani@polban.ac.id, \{ebaskoro, suhadi\}@math.itb.ac.id
}

\begin{abstract}
Burger and Vuuren defined the size multipartite Ramsey number for a pair of complete, balanced, multipartite graphs $m_{j}\left(K_{a \times b}, K_{c \times d}\right)$, for natural numbers $a, b, c, d$ and $j$, where $a, c \geq 2$, in 2004 . They have also determined the necessary and sufficient conditions for the existence of size multipartite Ramsey numbers $m_{j}\left(K_{a \times b}, K_{c \times d}\right)$. Syafrizal et. al. generalized this definition by removing the completeness requirement. For simple graphs $G$ and $H$, they defined the size multipartite Ramsey number $m_{j}(G, H)$ as the smallest natural number $t$ such that any red-blue coloring on the edges of $K_{j \times t}$ contains a red $G$ or a blue $H$ as a subgraph. In this paper, we determine the necessary and sufficient conditions for the existence of multipartite Ramsey numbers $m_{j}(G, H)$, where both $G$ and $H$ are non complete graphs. Furthermore, we determine the exact values of the size multipartite Ramsey numbers $m_{j}\left(K_{1, m}, K_{1, n}\right)$ for all integers $m, n \geq 1$ and $j=2,3$, where $K_{1, m}$ is a star of order $m+1$. In addition, we also determine the lower bound of $m_{3}\left(k K_{1, m}, C_{3}\right)$, where $k K_{1, m}$ is a disjoint union of $k$ copies of a star $K_{1, m}$ and $C_{3}$ is a cycle of order 3 .
\end{abstract}

Keywords: cycle, existence, size multipartite Ramsey number, star.

Mathematics Subject Classification : 05C55

DOI: 10.19184/ijc.2019.3.2.4

\section{Introduction}

The classical Ramsey number $r(a, c)$ is the smallest natural number $j$ such that any red-blue coloring of the edges of $K_{j}$, necessarily forces a red $K_{a}$ or a blue $K_{c}$ as subgraph. The size multipartite Ramsey number is one of generalizations of the classical Ramsey number. Burger and Vuuren [1] gave a definition of the size multipartite Ramsey numbers for a pair of complete, balanced, multipartite graphs, as follows. Let $a, b, c, d$ and $j$, be natural numbers with $a, c \geq 2$, the

Received: 6 Jul 2019, Revised: 25 Nov 2019, Accepted: 5 Dec 2019. 
size multipartite Ramsey number $m_{j}\left(K_{a \times b}, K_{c \times d}\right)$ is the smallest natural number $t$ such that any red-blue coloring of the edges of $K_{j \times t}$, necessarily forces a red $K_{a \times b}$ or a blue $K_{c \times d}$ as subgraph. They also determined $m_{j}\left(K_{2 \times 2}, K_{3 \times 1}\right)$, for $j \geq 1$ and have established the following existence of size multipartite Ramsey numbers.

Theorem 1.1. (The existence of size numbers) [1]

The size multipartite Ramsey numbers $m_{j}\left(K_{a \times b}, K_{c \times d}\right)$ exists for any $a, c \geq 2$ and $b, d \geq 1$ if and only if $j \geq r(a, c)$.

Syafrizal et. al. [10] generalized this definition by removing the completeness requirement. For simple graphs $G$ and $H$, they defined the size multipartite Ramsey number $m_{j}(G, H)$ as the smallest natural number $t$ such that any red-blue coloring on the edges of $K_{j \times t}$ contains a red $G$ or a blue $H$ as a subgraph. The size bipartite Ramsey numbers for stars versus paths $m_{2}\left(K_{1, m}, P_{n}\right)$, for $m, n \geq 2$ given by Hattingh and Henning [3]. In 2007, Syafrizal et al. [11] determined the size multipartite Ramsey numbers for stars versus $P_{3}$. Then, Surahmat et al. [9] gave the size tripartite Ramsey numbers for stars versus $P_{n}$, for $3 \leq n \leq 6$. Furthermore, we gave the size multipartite Ramsey numbers for stars versus cycles [5] and the size tripartite Ramsey numbers for a disjoint union of $m$ copies of a star $K_{1, n}$ versus $P_{3}$ [6]. In 2017, Jayawardene et al. [4] and Effendi et al. [2] determined the size multipartite Ramsey numbers for stars versus paths. Then, we also gave the size multipartite Ramsey numbers for stars versus paths and cycles [7], that complete the previous results given by Syafrizal and Surahmat. Recently, we determined $m_{j}\left(m K_{1, n}, H\right)$, where $H=P_{3}$ or $K_{1,3}$ for $j \geq 3, m, n \geq 2$ [8].

In this paper, we determine the necessary and sufficient conditions for the existence of the size multipartite Ramsey numbers $m_{j}(G, H)$, where both $G$ and $H$ are non complete graphs. Furthermore, we determine the exact values of the size multipartite Ramsey numbers $m_{j}\left(K_{1, m}, K_{1, n}\right)$ for all integers $m, n \geq 1$ and $j=2,3$. In addition, we also determine the lower bound of $m_{3}\left(k K_{1, m}, C_{3}\right)$.

We call some basic definitions that will be used in this paper, as follows. Let $G$ be a finite and simple graph. Let vertex and edge sets of graph $\mathrm{G}$ are denoted by $V(G)$ and $E(G)$, respectively. Vertex colorings in which adjacent vertices are colored differently are proper vertex colorings. A graph $G$ is $k$-colorable if there exists a proper vertex coloring of $G$ from a set of $k$ colors. A matching of a graph $G$ is defined as a set of edges without a common vertex. A matching of maximum size in $G$ is a maximum matching in $G$. The maximum degree of $G$ is denoted by $\Delta(G)$, where $\Delta(G)=\max \{d(v) \mid v \in V(G)\}$. The minimum degree of $G$ is denoted by $\delta(G)$, where $\delta(G)=\min \{d(v) \mid v \in V(G)\}$. A star $K_{1, n}$ is the graph on $n+1$ vertices with one vertex of degree $n$, called the center of this star, and $n$ vertices of degree 1 , called the leaves. A disjoint union of $k$ copies of a star $K_{1, m}$, a cycle of order $n$, and a path of order $n$ are denoted by $k K_{1, m}, C_{n}$, and $P_{n}$, respectively.

\section{Results}

For any non complete graphs $G$ and $H$, we will determine the necessary and sufficient conditions for the existence of the size multipartite Ramsey numbers $m_{j}(G, H)$. In order to do so, 
we recall the definition of the chromatic number of a graph $G$, denoted by $\chi(G)$, which is the minimum positive integer $k$ for which $G$ is $k$-colorable.

Lemma 2.1. In every proper vertex coloring of a simple graph $G$, the maximum number of the vertices in $G$ with the same color is $|V(G)|-\chi(G)+1$.

Proof. Let $c$ be a proper vertex coloring of $G$, with $\chi(G)$ color, that is $c: V(G) \rightarrow\{1,2, \ldots, \chi(G)\}$. Let $C_{i}=\{v \in V(G) \mid c(v)=i\}$. Without lost generality, let $\left|C_{1}\right| \leq\left|C_{2}\right| \leq \ldots \leq\left|C_{\chi(G)}\right|$. Since for $1 \leq i \leq \chi(G)-1$, we have $\left|C_{i}\right| \geq 1$, then $\left|C_{\chi(G)}\right| \leq|V(G)|-\chi(G)+1$.

Theorem 2.1. Let $G$ and $H$ be two non complete graph. The multipartite Ramsey numbers $m_{j}(G, H)$ are finite if and only if $j \geq \operatorname{maks}\{\chi(G), \chi(H)\}$.

Proof. Let $m_{j}(G, H)=t<\infty$, that is $K_{j \times t} \rightarrow(G, H)$. If $K_{j \times t}=F_{1} \oplus F_{2}$, then $\left(F_{1} \nsupseteq G \Rightarrow\right.$ $\left.F_{2} \supseteq H\right)$ or $\left(F_{2} \nsupseteq H \Rightarrow F_{1} \supseteq G\right)$. This implies that $j \geq \chi(H)$ and $\left.j \geq \chi(G)\right\}$. Therefore, $j \geq \operatorname{maks}\{\chi(G), \chi(H)\}$.

Let $j \geq \operatorname{maks}\{\chi(G), \chi(H)\}$. We show that $m_{j}(G, H)$ is finite. We construct an positive integer $t$ such that $K_{j \times t} \rightarrow(G, H)$. Let $p=|V(G)|-\chi(G)+1, q=|V(H)|-\chi(H)+1$ and $t=p+q$. Note that $V\left(K_{j \times t}\right)=V\left(K_{j \times p}\right) \cup V\left(K_{j \times q}\right)$. Based on Lemma 2.1, $p$ and $q$ are the maximum number of the same colored vertices in $G$ and $H$, respectively, so $K_{j \times p} \supseteq G$ and $K_{j \times q} \supseteq H$. Therefore, $K_{j \times t} \rightarrow(G, H)$. Then, $m_{j}(G, H) \leq t$. Since graph $G$ and $H$ are finite graph, so $|V(G)|,|V(H)|, \chi(G)$ and $\chi(H)$ are finite. So, $m_{j}(G, H) \leq t<\infty$. Then, $m_{j}(G, H)$ is finite.

Theorem 2.2. For positive integers $m$ and $n$, we have $m_{2}\left(K_{1, m}, K_{1, n}\right)=m+n-1$.

Proof. We will show that $m_{2}\left(K_{1, m}, K_{1, n}\right) \geq m+n-1$. We consider a red-blue coloring on the edges of graph $K_{2 \times(m+n-2)}=F_{R} \oplus F_{B}$, such that $F_{R}$ is a $(m-1)$-regular graph. By Handshaking Lemma, it is possible since the sum of the degrees of the vertices of $F_{R}$ is even. Then, $F_{R} \nsupseteq K_{1, m}$. We have $d(v)=m+n-2-(m-1)=n-1$, for any $v$ in $F_{B}$. Hence, $F_{B} \nsupseteq K_{1, n}$.

Now, we will show that $m_{2}\left(K_{1, m}, K_{1, n}\right) \leq m+n-1$. We consider any red-blue coloring on the edges of graph $K_{2 \times(m+n-1)}=G_{R} \oplus G_{B}$, such that $G_{R} \nsupseteq K_{1, m}$. This implies that $\Delta\left(G_{R}\right) \leq m-1$. Therefore, $\delta\left(G_{B}\right) \geq m+n-1-(m-1)=n$. Then, $G_{B} \supseteq K_{1, n}$.

Theorem 2.3. For positive integers $m$ and $n$, we have

$$
m_{3}\left(K_{1, m}, K_{1, n}\right)= \begin{cases}\frac{m}{2}, & \text { for } m \equiv 2 \bmod 4, n=1,2 \\ 2\left\lfloor\frac{m+1}{4}\right\rfloor+2\left\lceil\frac{n}{4}\right\rceil, & \text { for } m \equiv 2 \bmod 4, n \equiv 3 \bmod 4, \\ 2\left\lfloor\frac{m-1}{4}\right\rfloor+2\left\lceil\frac{n}{4}\right\rceil, & \text { for } m \equiv 4 \bmod 4, n \equiv 1 \bmod 4, \\ \frac{m-1}{2}+\left\lceil\frac{n}{2}\right\rceil, & \text { for } m \equiv 1 \bmod 2, n \geq 1, \\ 2\left\lfloor\frac{m+1}{4}\right\rfloor+2\left\lfloor\frac{n}{4}\right\rfloor+1, & \text { for } m \equiv 2 \bmod 4, n \neq 3 \bmod 4, n \geq 4, \\ 2\left\lfloor\frac{m-1}{4}\right\rfloor+2\left\lceil\frac{n}{4}\right\rceil+1, & \text { for } m \equiv 4 \bmod 4, n \neq 1 \bmod 4 .\end{cases}
$$

Proof. Case 1. $m_{3}\left(K_{1, m}, K_{1, n}\right)=\frac{m}{2}$, for $m \equiv 2 \bmod 4$, and $n=1,2$. 
For $n=1$, we will use the property that $m_{3}\left(K_{1, m}, K_{1}\right) \leq m_{3}\left(K_{1, m}, K_{1,1}\right)$. It is clear that $m_{3}\left(K_{1, m}, K_{1}\right)=\frac{m}{2}$. Therefore, $m_{3}\left(K_{1, m}, K_{1,1}\right) \geq \frac{m}{2}$. If $K_{3 \times \frac{m}{2}}$ contains no a blue $K_{1,1}$, then $K_{3 \times \frac{m}{2}}$ contains a red $K_{1, m}$, since $d(v)=m$, for any $v$ in $K_{3 \times \frac{m}{2}}$. Hence, $m_{3}\left(K_{1, m}, K_{1,1}\right) \leq \frac{m}{2}$.

For $m=n=2$, it is clear that $m_{3}\left(K_{1, m}, K_{1, n}\right) \geq \frac{m}{2}$. For $m \equiv 6 \bmod 4$ and $n=2$, we consider a red-blue coloring on the edges of graph $K_{3 \times\left(\frac{m}{2}-1\right)}$, such that $K_{3 \times\left(\frac{m}{2}-1\right)}$ contains a maximum blue matching graph. Since $\frac{m}{2}-1$ is even, the blue graph is a 1 -regular graph. This implies that graph $K_{3 \times\left(\frac{m}{2}-1\right)}$ contains red $(m-3)$-regular graph. So $K_{3 \times\left(\frac{m}{2}-1\right)}$ contains no a red $K_{1, m}$. Then, $m_{3}\left(K_{1, m}, K_{1,2}\right) \geq \frac{m}{2}$. Furthermore, we consider any red-blue coloring on the edges of graph $K_{3 \times \frac{m}{2}}$, such that graph $K_{3 \times \frac{m}{2}}$ contains no a blue $K_{1,2}$. This implies that the maximum degree of blue graph is 1 . Since $\frac{m}{2}$ is odd, then there is at least one vertex $v$, where $d(v)=0$ in blue graph and $d(v)=m$ in red graph. Then, $K_{3 \times \frac{m}{2}}$ contains a red $K_{1, m}$. Therefore, $m_{3}\left(K_{1, m}, K_{1,2}\right) \leq \frac{m}{2}$.

Case 2. For $(m \equiv 2 \bmod 4$ and $n \equiv 3 \bmod 4)$, let $t=2\left\lfloor\frac{m+1}{4}\right\rfloor+2\left\lceil\frac{n}{4}\right\rceil$ and for $(m \equiv 4 \bmod 4$ and $n \equiv 1 \bmod 4)$, let $t=2\left\lfloor\frac{m-1}{4}\right\rfloor+2\left\lceil\frac{n}{4}\right\rceil$.

We consider a red-blue coloring on the edges of graph $K_{3 \times(t-1)}=F_{R} \oplus F_{B}$, such that $d\left(v_{1}\right)=$ $m-2$, for a vertex $v_{1} \in V\left(F_{R}\right)$ and $d(v)=m-1$, for any $v \in V\left(F_{R}\right)-\left\{v_{1}\right\}$. By Handshaking Lemma, it is possible since the sum of the degrees of the vertices of $F_{R}$ is even. Then, $F_{R} \nsupseteq K_{1, m}$. We distinguish the following two cases, to show that $m_{3}\left(K_{1, m}, K_{1, n}\right) \geq t$.

Case a. For $m \equiv 2 \bmod 4$ and $n \equiv 3 \bmod 4$.

We have $d\left(v_{1}\right)=2 t-m=4\left\lfloor\frac{m+1}{4}\right\rfloor+4\left\lceil\frac{n}{4}\right\rceil-m=m-2+n+1-m=n-1$, for $v_{1} \in V\left(F_{B}\right)$ and $d(v)=2 t-m-1=4\left\lfloor\frac{m+1}{4}\right\rfloor+4\left\lceil\frac{n}{4}\right\rceil-m-1=m-2+n+1-m-1=n-2$, for any $v \in V\left(F_{B}\right)-\left\{v_{1}\right\}$. Then, $F_{B} \nsupseteq K_{1, n}$.

Case b. For $m \equiv 4 \bmod 4$ and $n \equiv 1 \bmod 4$.

We have $d\left(v_{1}\right)=2 t-m=4\left\lfloor\frac{m-1}{4}\right\rfloor+4\left\lceil\frac{n}{4}\right\rceil-m=m-4+n+3-m=n-1$, for $v_{1} \in V\left(F_{B}\right)$ and $d(v)=2 t-m-1=4\left\lfloor\frac{m+1}{4}\right\rfloor+4\left\lceil\frac{n}{4}\right\rceil-m-1=m-4+n+3-m-1=n-2$, for any $v \in V\left(F_{B}\right)-\left\{v_{1}\right\}$. Then, $F_{B} \nsupseteq K_{1, n}$.

Now, we consider any red-blue coloring on the edges of graph $K_{3 \times t}=G_{R} \oplus G_{B}$, such that $G_{R} \nsupseteq K_{1, m}$. This implies that $\Delta\left(G_{R}\right) \leq m-1$. We distinguish the following two cases, to show that $m_{3}\left(K_{1, m}, K_{1, n}\right) \leq t$.

Case a. For $m \equiv 2 \bmod 4$ and $n \equiv 3 \bmod 4$.

$\delta\left(G_{B}\right) \geq 2 t-(m-1)=2 t-m+1=m-1+2\left\lceil\frac{n}{2}\right\rceil-m+1=n+1$, since $n$ is odd. Then, $G_{B} \supseteq K_{1, n}$.

Case b. For $m \equiv 4 \bmod 4$ and $n \equiv 1 \bmod 4$.

$\delta\left(G_{B}\right) \geq 2 t-(m-1)=2 t-m+1=4\left\lfloor\frac{m-1}{4}\right\rfloor+4\left\lceil\frac{n}{4}\right\rceil-m+1=m-4+n+3-m+2=n$. Therefore, $G_{B} \supseteq K_{1, n}$.

Case 3. For $m \equiv 1 \bmod 2$ and $n \geq 1$, let $t=\frac{m-1}{2}+\left\lceil\frac{n}{2}\right\rceil$, for $m \equiv 2 \bmod 4$ and $n \neq 3 \bmod 4$, let $t=2\left\lfloor\frac{m+1}{4}\right\rfloor+2\left\lfloor\frac{n}{4}\right\rfloor+1$, and for $m \equiv 4 \bmod 4$ and $n \neq 1 \bmod 4$, let $t=2\left\lfloor\frac{m-1}{4}\right\rfloor+2\left\lceil\frac{n}{4}\right\rceil+1$.

We consider a red-blue coloring on the edges of graph $K_{3 \times(t-1)}=F_{R} \oplus F_{B}$, such that $F_{R}$ is a $(m-1)$-regular graph. By Handshaking Lemma, it is possible since the sum of the degrees of the vertices of $F_{R}$ is even. Then, $F_{R} \nsupseteq K_{1, m}$. We have $d(v)=2(t-1)-(m-1)$. We distinguish the following three cases, to show that $m_{3}\left(K_{1, m}, K_{1, n}\right) \geq t$. 
Case a. For $m \equiv 1 \bmod 2$ dan $n \geq 1$.

$d(v)=2 t-m-1=m-1+2\left\lceil\frac{n}{2}\right\rceil-m-1=2\left\lceil\frac{n}{2}\right\rceil-2<n$, for any $v$ in $F_{B}$. Then, $F_{B} \nsupseteq K_{1, n}$.

Case b. For $m \equiv 2 \bmod 4$ and $n \neq 3 \bmod 4$.

$d(v)=2 t-m-1=4\left\lfloor\frac{m+1}{4}\right\rfloor+4\left\lfloor\frac{n}{4}\right\rfloor+2-m-1=m-2+4\left\lfloor\frac{n}{4}\right\rfloor-m+1=4\left\lfloor\frac{n}{4}\right\rfloor-1 \leq n-1$, for any $v$ in $F_{B}$. Then, $F_{B} \nsupseteq K_{1, n}$.

Case c. For $m \equiv 4 \bmod 4$ and $n \neq 1 \bmod 4$.

$d(v)=2 t-m-1=4\left\lfloor\frac{m-1}{4}\right\rfloor+4\left\lceil\frac{n}{4}\right\rceil+2-m-1=m-4+4\left\lceil\frac{n}{4}\right\rceil-m+1=4\left\lceil\frac{n}{4}\right\rceil-3<n$, for any $v$ in $F_{B}$. Then, $F_{B} \nsupseteq K_{1, n}$.

Now, we consider any red-blue coloring on the edges of graph $K_{3 \times t}=G_{R} \oplus G_{B}$, such that $G_{R} \nsupseteq K_{1, m}$. This implies that $\Delta\left(G_{R}\right) \leq m-1$. We distinguish the following three cases, to show that $m_{3}\left(K_{1, m}, K_{1, n}\right) \leq t$.

Case a. For $m \equiv 1 \bmod 2$ dan $n \geq 1$.

$\delta\left(G_{B}\right) \geq 2 t-(m-1)=2 t-m+1=m-1+2\left\lceil\frac{n}{2}\right\rceil-m+1=2\left\lceil\frac{n}{2}\right\rceil \geq n$. Then, $G_{B} \supseteq K_{1, n}$.

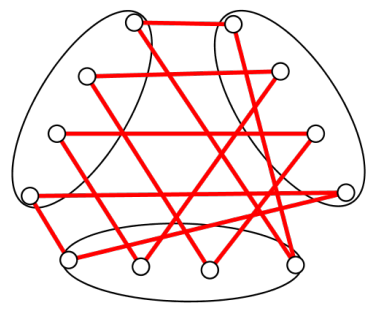

$G_{R} \nsupseteq K_{1,3}$

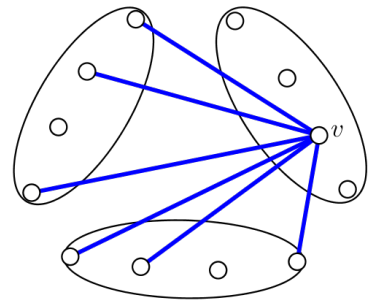

$G_{B} \supseteq K_{1,6}$

Figure 1. A coloring for $m_{3}\left(K_{1,3}, K_{1,6}\right)=4$.

For $m$ and $n$ are both even, suppose that $d(v)=m-1$, for any $v$ in $G_{R}$. Then, the sum of the degrees of the vertices of $G_{R}$ is odd. By Handshaking Lemma, it is a contradiction. Then, there is at least one vertex $v_{1}$ in $G_{R}$ such that $d\left(v_{1}\right)=m-2$. We consider $v_{1}$ in $G_{B}$ for the following two cases.

Case b. For $m \equiv 2 \bmod 4$ and $n \neq 3 \bmod 4$.

$d\left(v_{1}\right)=2 t-m+2=4\left\lfloor\frac{m+1}{4}\right\rfloor+4\left\lfloor\frac{n}{4}\right\rfloor+2-m+2=m-2+4\left\lfloor\frac{n}{4}\right\rfloor-m+4=4\left\lfloor\frac{n}{4}\right\rfloor+2 \geq n$. Case c. For $m \equiv 4 \bmod 4$ and $n \neq 1 \bmod 4$.

$d\left(v_{1}\right)=2 t-m+2=4\left\lfloor\frac{m-1}{4}\right\rfloor+4\left\lceil\frac{n}{4}\right\rceil+2-m+2=m-4+4\left\lceil\frac{n}{4}\right\rceil-m+4=4\left\lceil\frac{n}{4}\right\rceil \geq n$. Therefore, there is a star $K_{1, n}$ in $G_{B}$, where $v_{1}$ as the center. 


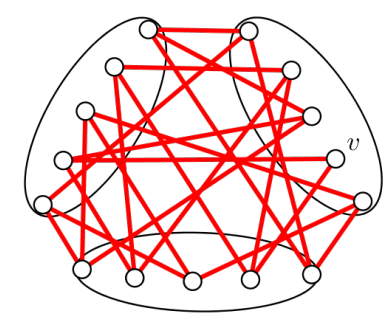

$G_{R} \nsupseteq K_{1,4}$

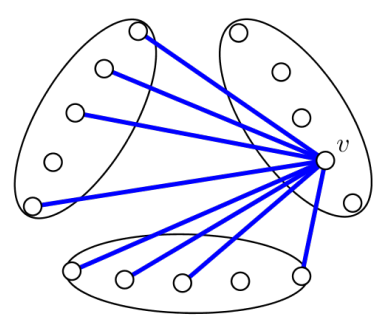

$G_{B} \supseteq K_{1, n}(6 \leq n \leq 8)$

Figure 2. A coloring for $m_{3}\left(K_{1,4}, K_{1, n}\right)=5,(6 \leq n \leq 8)$.

Theorem 2.4. For positive integers $m$ and $n$, we have

$$
m_{3}\left(m K_{1, n}, C_{3}\right) \geq n\left\lceil\frac{m}{2}\right\rceil+\left\lfloor\frac{m}{2}\right\rfloor .
$$

Proof. Let $t=n\left\lceil\frac{m}{2}\right\rceil+\left\lfloor\frac{m}{2}\right\rfloor$. We will show that $m_{3}\left(m K_{1, n}, C_{3}\right) \geq t$. Let $A, B$ and $C$ be three partite sets in graph $K_{3 \times(t-1)}$. We consider a red-blue coloring on the edges of graph $K_{3 \times(t-1)}=$ $F_{R} \oplus F_{B}$ such that $F_{B}=K_{t-1,2(t-1)}$, where the first partite set is $A$ and the second partite set is $B \cup C$. This implies that $F_{R}=K_{2 \times(t-1)}$, where the partite sets are $B$ and $C$. If $m$ is even, then $\left|V\left(F_{R}\right)\right|=2(t-1)=2\left(n\left\lceil\frac{m}{2}\right\rceil+\left\lfloor\frac{m}{2}\right\rfloor-1\right)=m(n+1)-2<\left|V\left(m K_{1, n}\right)\right|$. Therefore, $F_{R} \nsupseteq m K_{1, n}$. If $m=1$, then $F_{R}=K_{2 \times(n-1)}$. It is clear that $F_{R} \nsupseteq K_{1, n}$. If $m \geq 3$ and $m$ is odd, then $|B|=|C|=\frac{n(m+1)}{2}+\frac{m-3}{2}=\frac{m-1}{2}(n+1)+\frac{n-1}{2}$. Hence, $F_{R}$ only contains $(m-1) K_{1, n}$. Then, $m_{3}\left(m K_{1, n}, C_{3}\right) \geq t$.

\section{Acknowledgement}

This research was supported by Research Grant "Penelitian Mandiri" Surat Keputusan No. 438.68/PL1.R7/LT/2019, Politeknik Negeri Bandung, Indonesia.

\section{References}

[1] A. P. Burger and J. H. van Vuuren, Ramsey numbers in complete balanced multipartite graphs Part II: Size Numbers, Discrete Math. 283 (2004), 45-49.

[2] Effendi, A. I. Baqi, and Syafrizal Sy, On size multipartite Ramsey numbers for paths versus stars, Int. J. Math. Analysis 10 (2016), 1061-1065.

[3] J. H. Hattingh and M. A. Henning, Star-path bipartite Ramsey numbers, Discrete Math. 185 (1998), 255-258.

[4] C. Jayawardene and L. Samarasekara, A strict upper bound for size multipartite Ramsey numbers of paths versus stars, Indones. J. Combin. 1 (2) (2017), 55-63.

[5] A. Lusiani, Syafrizal Sy, E. T. Baskoro, and C. Jayawardene, On size multipartite Ramsey numbers for stars versus cycles, Procedia Comput. Sci. 74 (2015), 27-31. 
[6] A. Lusiani, E. T. Baskoro, and S. W. Saputro, On size tripartite Ramsey numbers of $P_{3}$ versus $m K_{1, n}$, AIP. Conf. Proc. 1707, 020010 (2016), doi:10.1063/1.4940811.

[7] A. Lusiani, E. T. Baskoro, and S. W. Saputro, On size multipartite Ramsey numbers for stars versus paths and cycles, Electron. J. Graph Theory Appl. 5 (1) (2017), 43-50.

[8] A. Lusiani, E. T. Baskoro, and S. W. Saputro, On size multipartite Ramsey numbers of $m K_{1, n}$ versus $P_{3}$ and $K_{1,3}$, Proc. Jangjeon Math. Soc. 22 (1) (2019), 59-65, doi:10.17777/pjms2019.22.1.59.

[9] Surahmat and Syafrizal Sy, Star-path size multipartite Ramsey numbers, Appl. Math. Sci. (Bulgaria) 8 (75) (2014), 3733-3736.

[10] Syafrizal Sy, E. T. Baskoro, and S. Uttunggadewa, The size multipartite Ramsey number for paths, J. Combin. Math. Combin. Comput. 55 (2005), 103-107.

[11] Syafrizal Sy, E. T. Baskoro, and S. Uttunggadewa, The size multipartite Ramsey numbers for small paths versus other graphs, Far East J. Appl. Math. 28 (1) (2007), 131-138. 